ELECTRONIC RESEARCH ANNOUNCEMENTS OF THE AMERICAN MATHEMATICAL SOCIETY

Volume 3, Pages 17-27 (April 8, 1997)

S $1079-6762(97) 00019-\mathrm{X}$

\title{
POLYNOMIALS WITH INTEGRAL COEFFICIENTS, EQUIVALENT TO A GIVEN POLYNOMIAL
}

\author{
JÁNOS KOLLÁR
}

(Communicated by Robert Lazarsfeld)

\begin{abstract}
Let $f\left(x_{0}, \ldots, x_{n}\right)$ be a homogeneous polynomial with rational coefficients. The aim of this paper is to find a polynomial with integral coefficients $F\left(x_{0}, \ldots, x_{n}\right)$ which is "equivalent" to $f$ and as "simple" as possible. The principal ingredient of the proof is to connect this question with the geometric invariant theory of polynomials. Applications to binary forms, class numbers, quadratic forms and to families of cubic surfaces are given at the end.
\end{abstract}

\section{INTRODUCTION}

Let $f\left(x_{0}, \ldots, x_{n}\right)$ be a homogeneous polynomial with rational coefficients. The aim of this paper is to find a polynomial with integral coefficients $F\left(x_{0}, \ldots, x_{n}\right)$ which is "equivalent" to $f$ and as "simple" as possible.

There are two frequently considered notions of equivalence for polynomials. We say that two polynomials $f, g$ are affine equivalent if there is an invertible matrix $M \in G L(n+1, \mathbb{Q})$ such that $f(\mathbf{x})=g(M \mathbf{x}) . \quad f$ and $g$ are called projectively equivalent if there is an invertible matrix $M \in G L(n+1, \mathbb{Q})$ and a nonzero constant $c \in \mathbb{Q}^{*}$ such that $f(\mathbf{x})=c \cdot g(M \mathbf{x})$. Both of these concepts are equivalence relations which preserve the degree.

It is much less clear how to define which polynomials are "simple". In fact, the main aim of the present paper is to develop such a definition.

Many special cases of this question have been considered in the past.

1.1. The study of quadratic number fields $K=\mathbb{Q}(\sqrt{D})$ is intimately connected with the study of binary quadratic forms $Q=a x^{2}+b x y+c y^{2}$. Let $d_{K}$ denote the discriminant of $K$. A quadratic form $Q$ is called fundamental for $K$ if $b^{2}-4 a c=d_{K}$. The study of equivalence classes of fundamental quadratic forms is equivalent to the study of the ideal class group of $K$; see, for instance, [3, VII.2].

1.2. In the theory of elliptic curves it is frequently useful to look at a Weierstrass equation

$$
y^{2} z+a_{1} x y z+a_{3} y z^{2}=x^{3}+a_{2} x^{2} z+a_{4} x z^{2}+a_{6} z^{3}, \quad \text { where } a_{i} \in \mathbb{Z} .
$$

Received by the editors January 30, 1997.

1991 Mathematics Subject Classification. Primary 11G35, 14G25, 14D10; Secondary 11C08, 11E12, 11E76, 11R29, 14D25, 14J70.

Key words and phrases. Polynomials, hypersurfaces, geometric invariant theory, class numbers, quadratic forms.

(C) 1997 American Mathematical Society 
Such an equation is called minimal if its discriminant is the smallest possible; see [10, VIII.8] for details. (If $a_{1}=a_{2}=a_{3}=0$, then the discriminant is the usual expression $4 a_{4}^{3}+27 a_{6}^{2}$, up to a normalizing factor. The general form is quite complicated but it can be reduced to this one by suitable substitutions.)

1.3. In the fifties Demyanov, Lewis, and Springer studied the solvability of equations $f=0$ in the $p$-adic field $\mathbb{Q}_{p}$ (Artin's conjecture). Their method was to find an equivalent equation $F=0$ with coefficients in the $p$-adic integers $\mathbb{Z}_{p}$ such that the reduction of $F$ modulo $p$ is relatively simple. Their main concern was to ensure that $F$ modulo $p$ is irreducible, even over the algebraic closure of the finite field $\mathbb{F}_{p}$. See [7] for an overview and references.

1.4. Recently, Corti [1] studied families of Del Pezzo surfaces over smooth curves. His aim was to use birational transformations to obtain a family whose total space has very mild singularities. For cubic surfaces over Dedekind domains he proposed a program to obtain such a model. He proved that the program works over curves in characteristic zero, but the positive characteristic and the arithmetic cases were left open.

The intention of this paper is to propose a unified generalization of the above special cases.

1.5. Definition. Let $f(\mathbf{x}) \in \mathbb{Q}\left[x_{0}, \ldots, x_{n}\right]$ be a homogeneous polynomial. For any $M \in G L(n+1, \mathbb{Q})$ and $c \in \mathbb{Q}^{*}$ consider the new polynomial obtained by the coordinate change $F(\mathbf{x}):=c \cdot f(M \mathbf{x})$. It may happen that $F(\mathbf{x})$ has integer coefficients, in which case I call $F$ an integral model of $f$. If $F(\mathbf{x})$ is an integral model of $f$ and $A \in G L(n+1, \mathbb{Z})$, then $\pm F(A \mathbf{x})$ is also an integral model. Such integral models will be considered integrally equivalent.

The "simplest" integral models will be called semi-stable over $\mathbb{Z}$. (The choice of terminology should become clear later.) Before giving the precise definition (see (3.3)), I list the main properties that such a concept should possess.

1.6. The requirements for the theory. Semi-stable models over $\mathbb{Z}$ should satisfy the following properties:

(1.6.1) Being semi-stable over $\mathbb{Z}$ should be a local property. That is, $F(\mathbf{x})$ is semi-stable over $\mathbb{Z}$ iff it is semi-stable over $\mathbb{Z}_{p}$ (the ring of $p$-adic integers) for every $p$.

(1.6.2) $f$ should have only finitely many integral models (up to integral equivalence) which are semi-stable over $\mathbb{Z}$.

(1.6.3) Let $\Phi:$ (polynomials) $\rightarrow \mathbb{R}^{+}$be any function which is invariant under coordinate changes by $S L$ and satisfies $\Phi(c f)=c^{r} \Phi(f)$ for some $r=r(\Phi)>0$. Then an integral model $F$ of $f$ is semi-stable over $\mathbb{Z}$ iff $\Phi(F)$ is minimal among all integral models of $f$.

A procedure to construct semi-stable models for a polynomial over $\mathbb{Z}$ is described in (4.3). In some cases this procedure does not produce integral models satisfying all of the above properties (4.4). On the other hand, such polynomials turn out to be rare, as shown by the following special case of the main theorem (4.1):

1.7. Theorem. Let $f(\mathbf{x}) \in \mathbb{Q}\left[x_{0}, \ldots, x_{n}\right]$ be a homogeneous polynomial and assume that $(f=0) \subset \mathbb{C P}^{n}$ is a smooth hypersurface. Then $f$ has semi-stable models over $\mathbb{Z}$ which satisfy the properties (1.6.1-3). 
A key point of the proof is to connect our problem with the Hilbert-Mumford geometric invariant theory of hypersurfaces, revealing the correct class of polynomials for which semi-stable models over $\mathbb{Z}$ can be defined.

1.8. Generalizations. Instead of $\mathbb{Z}$, one can work over other rings. There are no major changes for principal ideal domains. It seems to me that the natural setting of the construction is over arbitrary Dedekind domains, or over one-dimensional regular schemes. In these cases we obtain polynomials defined on vector bundles.

One can try to work over an arbitrary normal scheme, but, as far as I can tell, the theory is restricted to codimension one primes.

The most general setting is probably the following. Let $C$ be a one-dimensional regular scheme and $G / C$ a group scheme over $C$ which acts on a scheme $X / C$. Given a closed point $P$ of the generic fiber of $X / C$, we want to find a "simple" section $s: C \rightarrow X$ which passes through the $G$-orbit of $P$. (If $G=G L$ and $X$ is a symmetric power of its standard representation, we obtain the case of homogeneous polynomials considered above.)

1.9. What is missing? In this paper we consider integral models which are "simple" at every prime $p$. It is very natural to ask for a theory which also takes into account the infinite prime. For definite quadratic forms such an approach is provided by the reduction theory of Minkowski-Siegel. Unfortunately, I do not know how to generalize it to higher degree polynomials.

It is also not clear that the simplest integral model of a polynomial should be a polynomial. This is more apparent if we look at the corresponding hypersurface $X_{\mathbb{Q}}$ over $\mathbb{Q}$ instead. This can be extended to a scheme $X_{\mathbb{Z}}$ in many different ways. In many cases it is advantageous to consider a model $X_{\mathbb{Z}}$ which is not a hypersurface. One such example is in [5, V.5].

One can also ask the more general question: given a scheme over $\mathbb{Q}$, how can we extend it to a scheme over $\mathbb{Z}$ in the simplest way? This is closely related to the general geometric invariant theory problem considered in (1.8).

Finally, it would be desirable to have an algorithm which constructs semi-stable models. The proof of the existence of semi-stable models (4.1) is very close to being algorithmic, but so far I could not prove a bound on the weights occurring in (4.3), except in some special cases.

\section{ACKNOWLEDGEMENTS}

I would like to thank A. Bertram, H. Clemens, J.-L. Colliot-Thélène, S. Gersten, J. Harris, and R. Lazarsfeld for useful conversations and e-mails. Partial financial support was provided by the NSF under grant number DMS-9622394. These notes were typeset by $\mathcal{A}_{\mathcal{M}} \mathcal{S}$ - $\mathrm{TE}_{\mathrm{E}} \mathrm{X}$, the $\mathrm{T}_{\mathrm{EX}}$ macro system of the American Mathematical Society.

\section{A SHORT REVIEW OF GEOMETRIC INVARIANT THEORY}

2.1. Definition. Let $S$ be a ring. A weight system $(\mathbf{x}, \mathbf{w})$ on $S\left[y_{0}, \ldots, y_{n}\right]$ is a choice of coordinates

$$
\left(x_{0}, \ldots, x_{n}\right)^{t}=M\left(y_{0}, \ldots, y_{n}\right)^{t}, \quad \text { where } \quad M \in S L(n+1, S),
$$

and weights $x_{i} \mapsto w_{i}:=w\left(x_{i}\right) \in \mathbb{R}$. (I frequently write this in vector notation as $\mathbf{x}=M \mathbf{y}$.) 
It may be more appropriate to allow $M \in G L(n+1, S)$. At the end this leads to an equivalent theory, but intermediate steps become more complicated since strong approximation (cf. [4, Ch.V]) does not hold for $G L$.

A weight system is called trivial if all the $w_{i}$ are the same.

Let $t$ be a new variable. For $f \in S\left[y_{0}, \ldots, y_{n}\right]$ set

$$
f\left(t^{\mathbf{w}} \mathbf{x}\right):=f\left(t^{w_{0}} x_{0}, \ldots, t^{w_{n}} x_{n}\right) .
$$

The multiplicity of $f$ at $(\mathbf{x}, \mathbf{w})$ is defined as the exponent of the lowest $t$-power occurring in $f\left(t^{\mathbf{w}} \mathbf{x}\right)$. It is denoted by mult $t f\left(t^{\mathbf{w}} \mathbf{x}\right)$.

2.2. Definition. Let $K$ be a field and $f \in K\left[y_{0}, \ldots, y_{n}\right]$ a homogeneous polynomial. Let $X:=(f=0) \subset \mathbb{P}_{K}^{n}$ be the corresponding hypersurface. A weight system $(\mathbf{x}, \mathbf{w})$ over $K$ is called

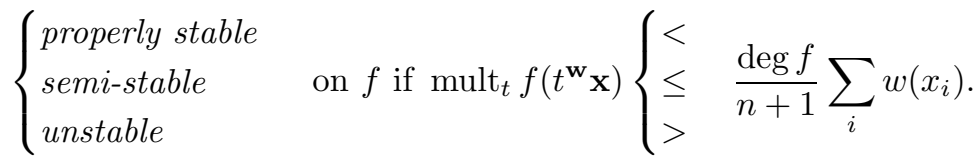

(The value on the right-hand side is the average multiplicity of a monomial in $f$. So unstable means roughly that more than half of the monomials are missing from f.)

These notions are invariant under affine linear changes of the weights $w_{i} \mapsto$ $a w_{i}+b$.

2.3. Definition. Let $K$ be a field with algebraic closure $\bar{K}$ and $f \in K\left[y_{0}, \ldots, y_{n}\right]$ a homogeneous polynomial. Let $X:=(f=0) \subset \mathbb{P}_{K}^{n}$ be the corresponding hypersurface.

(2.3.1) $f$ or $X$ is called properly stable (resp. semi-stable) iff every nontrivial weight system over $\bar{K}$ is properly stable (resp. semi-stable) on $f$. $f$.

(2.3.2) $f$ or $X$ is called unstable iff there is an unstable weight system over $\bar{K}$ on

For both of these cases it is sufficient to consider weight systems with integral weights.

2.4. Definition. Fix an infinite field $K$ and let $V_{d}$ denote the vector space of all degree $d$ homogeneous polynomials $f \in K\left[x_{0}, \ldots, x_{n}\right]$. $S L(n+1, K)$ acts by coordinate changes on $V_{d}$, thus it also acts on $K\left[V_{d}\right]$, the ring of all polynomials on $V_{d}$.

Any invarant $I \in K\left[V_{d}\right]$ of this action is called a polynomial $S L$-invariant of degree $d$ polynomials.

In general such invariants are hard to write down since they have very high degree. The best known invariant is the discriminant $D(f)$. By definition this is the unique (up to a multiplicative constant) lowest degree invariant such that $D(f)=0$ iff the corresponding hypersurface $(f=0)$ is singular (cf. [8]).

We need the following basic result (cf. [9, pp. 49 and 79]):

2.5. Theorem (Hilbert-Mumford criterion). Let $K$ be an infinite field and $f \in$ $K\left[x_{0}, \ldots, x_{n}\right]$ a degree $d$ homogeneous polynomial.

(2.5.1) There is a polynomial $S L$-invariant $I$ such that $I(f) \neq 0$ iff $f$ is semistable.

(2.5.2) If $(f=0)$ is a smooth hypersurface and $\operatorname{deg} f \geq 3$, then $f$ is properly stable. 


\section{The MAIN DEFinitions}

The theories using affine and projective equivalences are very similar, but I did not find any convenient way to treat them together. The following definitions all use projective equivalence, which is more natural from the point of view of algebraic geometry.

3.1. Notation. In this section $R$ denotes a principal ideal domain with quotient field $K$. Let $p \in R$ be a prime element and $k=R /(p)$ the residue field. For a polynomial $f_{R} \in R\left[y_{0}, \ldots, y_{n}\right], f_{k}$ denotes its reduction modulo $(p)$.

3.2. Definition. Notation as in (3.1). A weight system $(\mathbf{x}, \mathbf{w})$ is called integral if $w_{i} \in \mathbb{Z}$ for every $i$.

Let $f \in K\left[y_{0}, \ldots, y_{n}\right]$ be a polynomial. One can always find $s \in \mathbb{Z}$ and $p^{\prime} \in R$ prime to $p$ such that $p^{-s} \cdot p^{\prime} \cdot f \in R\left[y_{0}, \ldots, y_{n}\right]$. The largest such $s$ is called the multiplicty of $f$ at $p$; it is denoted by $\operatorname{mult}_{p} f$.

3.3. Definition. Notation as in (3.1). Let $f_{R} \in R\left[y_{0}, \ldots, y_{n}\right]$ be a homogeneous polynomial and $X_{R} \subset \mathbb{P}_{R}^{n}$ the hypersurface defined by the equation $\left(f_{R}=0\right)$.

(3.3.1) An integral weight system $(\mathbf{x}, \mathbf{w})$ over $R$ is called (projectively)

$$
\left\{\begin{array} { l } 
{ \text { properly stable } } \\
{ \text { semi-stable } } \\
{ \text { unstable } }
\end{array} \text { on } f _ { R } \text { at } p \text { if } \text { mult } _ { p } f _ { R } ( p ^ { \mathbf { w } } \mathbf { x } ) \left\{\begin{array}{l}
< \\
\leq \\
>
\end{array} \frac{\operatorname{deg} f_{R}}{n+1} \sum_{i} w_{i} .\right.\right.
$$

(3.3.2) $f_{R}$ (or $X_{R}$ ) is called properly stable (resp. semi-stable) at $p$ over $R$ if every weight system is properly stable (resp. semi-stable) on $f_{R}$ at $p$.

(3.3.3) $f_{R}$ (or $X_{R}$ ) is called unstable at $p$ over $R$ if there is an unstable weight system on $f_{R}$ at $p$

(3.3.4) $f_{R}$ (or $X_{R}$ ) is called properly stable (resp. semi-stable) over $R$ if it is properly stable (resp. semi-stable) at $p$ over $R$ for every prime $p \in R$.

(3.3.5) $f_{R}\left(\right.$ or $X_{R}$ ) is called unstable over $R$ if it is unstable at $p$ over $R$ for some $p$.

It is important to note that, unlike in $(2.2-2.3)$, here all coordinate changes must take place in $R$.

3.4. Comments. This is clearly a formal generalization of (2.3). In order to understand the precise relationship, let us consider the special case when $R=k[t]$, $p=t$ and $f_{R}=\sum_{J} a_{J}(t) x^{J}$.

Since the value of the weight of $t$ is fixed to be 1 , the other weights $w_{i}$ cannot be changed to $\lambda w_{i}$ without changing the concept.

It is nonetheless very useful to see what happens in the limits $\lambda \rightarrow 0, \infty$.

(3.4.1) If $\lambda \rightarrow 0$, then any monomial $a_{J}(t) x^{J}$ such that $t \mid a_{J}(t)$ becomes very large. Thus in the limit only the monomials with $t \not a_{J}(t)$ matter, that is, we are looking at the stability properties of $f_{k}$.

(3.4.2) If $\lambda \rightarrow \infty$, then mult $_{t} a_{J}(t)$ becomes very small compared with the $t$ powers coming from $x^{J}$. Thus in the limit only the vanishing or nonvanishing of the monomials matters, that is, we are looking at the stability properties of $f_{R}$ over $K$.

Thus (3.3) gives a notion that interpolates between the stability properties of the central and general fibers. 
3.5. Proposition. Notation as in (3.1).

(3.5.1) Assume that $f_{k} \in k\left[y_{0}, \ldots, y_{n}\right]$ is semi-stable (resp. properly stable) over $\bar{k}$. Then $f_{R}$ is semi-stable (resp. properly stable) at $p$.

(3.5.2) Let $\hat{R}$ denote the completion of $R$ at $(p)$. Then $f_{R} \in R[\mathbf{y}]$ is properly stable (semi-stable, unstable) at $p \in R$ iff $f_{R} \in \hat{R}[\mathbf{y}]$ is properly stable (semi-stable, unstable) at $p \in \hat{R}$.

Proof. The first part is easy. The second part is also clear once we note the following. By strong approximation (cf. [4, Ch.V]), the natural map $S L(n, R) \rightarrow$ $S L(n, \hat{R})$ has dense image, thus every weight system over $\hat{R}$ can be approximated by weight systems over $R$.

\section{The Main TheOREM OVER $\mathbb{Z}$}

The results of this section hold for affine and for projective equivalence as well. The first result settles the question of existence of semi-stable models.

4.1. Theorem. Let $f \in \mathbb{Q}\left[x_{0}, \ldots, x_{n}\right]$ be a homogeneous polynomial.

(4.1.1) $f$ has a semi-stable model over $\mathbb{Z}$ iff $f$ is semi-stable over $\mathbb{C}$.

(4.1.2) If $f$ is properly stable over $\mathbb{C}$, then $f$ has only finitely many semi-stable models over $\mathbb{Z}$ (up to the action of $S L(n+1, \mathbb{Z})$ ).

4.2. Remarks. If the stabilizer of $f$ in $G L(n+1, \mathbb{Q})$ is finite, then the converse of (4.1.2) also holds.

The implication $\Leftarrow$ of (4.1.1) holds if $\mathbb{Z}$ is replaced by any principal ideal domain $R$; the converse implication holds if char $R=0$.

(4.1.2) holds if all the residue fields of $R$ are finite.

The main part of the proof is the existence of semi-stable models over $\mathbb{Z}$.

4.3. A procedure to find semi-stable models. We start with a homogeneous polynomial $f_{\mathbb{Q}} \in \mathbb{Q}\left[y_{0}, \ldots, y_{n}\right]$.

Step 1: Find any $\mathbb{Z}$-model $F^{1}$ of $f_{\mathbb{Q}}$. For instance, $F^{1}:=N \cdot f_{\mathbb{Q}}$ will do for $N$ sufficiently divisible.

Step 2: Assume that we already have $F^{j}$. If $F^{j}$ is semi-stable at every prime $p$, then we are done.

Step 3: Otherwise there is a prime $p$ and an integral weight system $(\mathbf{x}, \mathbf{w})$ which is unstable on $F^{j}$. Set

$$
F^{j+1}:=p^{-s} F^{j}\left(p^{w_{0}} x_{0}, \ldots, p^{w_{n}} x_{n}\right), \quad \text { where } s:=\operatorname{mult}_{p} F^{j}\left(p^{\mathbf{w}} \mathbf{x}\right),
$$

and go back to Step 2 .

If the procedure ever stops, we obtain a semi-stable model of $f_{\mathbb{Q}}$. Unfortunately, in some cases the procedure never halts:

4.4. Example. For any prime $p$ consider the family of polynomials

$$
F_{i}:=p^{i} x_{0}^{4}+x_{2}\left(x_{0}^{3}+x_{1}^{3}\right) \quad \text { for } \quad i \geq 0 .
$$

Over $\mathbb{C}$ this gives an irreducible plane quartic curve with a triple point at $(0: 0: 1)$. Essentially the only unstable weight system at $p$ is given by the weights $w_{0}=w_{1}=$ $1, w_{2}=0$.

Applying Step 3 of (4.3) to $F_{i}$ yields $F_{i+1}$, thus we never reach a semi-stable model over $\mathbb{Z}$. In fact, one is tempted to say that among the above equations $F_{0}$ is 
the simplest, and our unsuccessful attempt to reach semi-stability results in more and more complicated equations.

A closer inspection of this example reveals that the problem stems from the fact that the $F_{i}$ are unstable over $\mathbb{C}$.

The proof that (4.3) halts in most cases, relies on the following lemma which was first used in this context by Laxton and Lewis [6]:

4.5. Lemma. Let $I(f)$ be a polynomial $S L$-invariant of degree $d$ polynomials in $n+1$ variables which has degree $r$ in the coefficients of $f$. If $M \in G L(n+1, \mathbb{Q})$ is a matrix and $c \in \mathbb{Q}$, then

$$
I(c \cdot f(M \mathbf{x}))=c^{r} \cdot(\operatorname{det} M)^{r d /(n+1)} I(f(\mathbf{x})) .
$$

In particular,

$$
I\left(p^{-s} f\left(p^{w_{0}} x_{0}, \ldots, p^{w_{n}} x_{n}\right)\right)=p^{r\left(-s+\frac{d}{n+1} \sum w_{i}\right)} I(f) .
$$

Proof. It is sufficient to check this over $\mathbb{C}$. There $M=c^{\prime} M^{\prime}$, where $M^{\prime} \in S L(n+$ $1, \mathbb{C}) . \quad I$ is invariant under $S L(n+1, \mathbb{C})$ and for scalar matrices the formula is clear.

4.6. Termination of the procedure. Assume now that $f$ is semi-stable. By (2.5) there is a polynomial $S L$-invariant $I$ such that $I(f) \neq 0$. We may assume that $I$ has integral coefficients, thus $I(F) \in \mathbb{Z}$ if $F \in \mathbb{Z}\left[x_{0}, \ldots, x_{n}\right]$.

By (4.5) we see that $I\left(F^{j+1}\right)$ is a proper divisor of $I\left(F^{j}\right)$; hence the procedure (4.3) will stop after finitely many steps.

The concept of proper stability over $\mathbb{Z}$ is connected with the uniqueness of semistable models. The following special case is the easiest to formulate:

4.7. Theorem. Let $F(\mathbf{y}) \in \mathbb{Z}\left[y_{0}, \ldots, y_{n}\right]$ be a homogeneous polynomial which is semi-stable over $\mathbb{Z}$. Assume that $\operatorname{deg} F$ and $n+1$ are relatively prime. The following are equivalent:

(4.7.1) $F(\mathbf{y})$ is properly stable over $\mathbb{Z}$.

(4.7.2) If $A \in G L(n+1, \mathbb{Q})$ is a matrix such that $F(A \mathbf{y})$ is semi-stable over $\mathbb{Z}$, then $A \in G L(n+1, \mathbb{Z})$.

Proof. Assume first that $F$ is not properly stable. Then there is a prime $p$ and a weight system $(\mathbf{x}=M \mathbf{y}, \mathbf{w})$ such that

$$
\operatorname{mult}_{p} F\left(p^{\mathbf{w}} \mathbf{x}\right)=\frac{\operatorname{deg} F}{n+1} \sum_{i} w_{i}
$$

(Equality holds since $F$ is semi-stable over $\mathbb{Z}$.) $\operatorname{deg} F$ divides mult $F\left(p^{\mathbf{w}} \mathbf{x}\right)$ since $\operatorname{deg} F$ and $n+1$ are relatively prime, thus we can introduce new weights

$$
w_{i}^{\prime}:=w_{i}-\frac{\operatorname{mult}_{p} F\left(p^{\mathbf{w}} \mathbf{x}\right)}{\operatorname{deg} F}, \text { so that } \sum w_{i}^{\prime}=0 .
$$

Set $A=\operatorname{diag}\left(p^{w_{0}^{\prime}}, \ldots, p^{w_{n}^{\prime}}\right) \cdot M$. Then $F(A \mathbf{x})$ is also semi-stable. $A \in G L(n+1, \mathbb{Z})$ iff $w_{i}^{\prime} \geq 0$ for every $i$. Since $\sum w_{i}^{\prime}=0$, this holds iff $w_{i}^{\prime}=0$ for every $i$, hence the weight system is trivial.

Conversely, assume that $F(\mathbf{y})$ is properly stable over $\mathbb{Z}$ and $F(A \mathbf{y})$ is semistable over $\mathbb{Z}$. By the theorem on elementary divisors (cf. $[12,12.2]$ ) we can write $A=M_{1} D M_{2}$, where $M_{i} \in S L(n+1, \mathbb{Z})$ and $D=\operatorname{diag}\left(r_{0}, \ldots, r_{n}\right)$ for some $r_{i} \in \mathbb{Q}^{*}$. If $A \notin G L(n+1, \mathbb{Z})$, then $r_{i} \notin \mathbb{Z}$ for some $i$, and we can construct a nontrivial 
weight system with weights $w_{i}:=$ mult $_{p} r_{i}$ for some $p$ which shows that $F$ is not properly stable. This contradiction shows (4.7).

Some standard results of geometric invariant theory and (3.5) imply the following result, which is an essential step in proving (4.1.2).

4.8. Theorem. Let $f(\mathbf{y}) \in \mathbb{Q}\left[y_{0}, \ldots, y_{n}\right]$ be a homogeneous polynomial which is properly stable over $\mathbb{C}$. Then $f$ is properly stable at $p$ for all but finitely many primes $p$.

\section{INTEGRAL MODELS OF HYPERSURFACES}

In this section I formulate the general versions of the theorems of the previous section.

Let $C$ be an integral, regular scheme of dimension 1 . Let $K$ denote the field of rational functions on $C$. If $p \in C$ is a closed point, then $\mathcal{O}_{p, C}$ denotes its local ring and $C_{p}:=\operatorname{Spec} \mathcal{O}_{p, C}$ the corresponding local scheme.

5.1. Theorem. Notation as above. Let $X_{K} \subset \mathbb{P}_{K}^{n}$ be a hypersurface and assume that $X_{K}$ is semi-stable (over $\bar{K}$ ).

Then there is a vector bundle $E$ of rank $n+1$ over $C$ and a hypersurface $X_{C} \subset$ $\mathbb{P}_{C}(E)$ such that

(5.1.1) The generic fiber of $X_{C}$ is isomorphic to $X_{K}$.

(5.1.2) For every closed point $p \in C$ the induced hypersurface $X_{C_{p}}$ is semi-stable over $\mathcal{O}_{p, C}$.

Any such $X_{C}$ is called a semi-stable model of $X_{K}$ over $C$.

Finiteness and uniqueness results hold in the properly stable case:

5.2. Theorem. Retain the above notation and assume that $X_{K}$ is properly stable (over $\bar{K})$. Let $X_{C}$ be a semi-stable model of $X_{K}$ over $C$. Then:

(5.2.1) There is a unique largest Zariski open set $C^{0}$, depending only on $X_{K}$, such that $X_{C}$ is properly stable at $p$ for every $p \in C^{0}$.

(5.2.2) If $X_{C}^{\prime}$ is another semi-stable model of $X_{K}$ over $C$, then $X_{C^{0}}^{\prime} \cong X_{C^{0}}$.

(5.2.3) If all residue fields of $C$ are finite, then $X_{K}$ has only finitely many semistable models over $C$ (up to isomorphism over $C$ ).

\section{EXAmples}

In this section we consider semi-stable models in various special cases.

6.1. Binary forms. The condition of semi-stability for binary forms over $\mathbb{Z}$ is especially simple. Over a field, an odd degree semi-stable form is properly stable, and the same unusual situation holds for binary forms over $\mathbb{Z}$ (or over any principal ideal domain):

6.1.1. Proposition. Let $F(x, y) \in \mathbb{Z}[x, y]$ be a homogeneous polynomial of degree d. $F$ is semi-stable (resp. properly stable) over $\mathbb{Z}$ iff $F$ is not divisible by any prime $p$ and the following holds:

$$
\left.\begin{array}{c}
p^{\frac{d+1}{2}} \\
p^{\frac{d}{2}}
\end{array}\right\} \backslash X F\left(\text { pax }+ \text { pby, cx+dy) } \forall \text { prime } p, \forall\left(\begin{array}{ll}
a & b \\
c & d
\end{array}\right) \in S L(2, \mathbb{Z}) .\right.
$$


6.1.2. Theorem. Let $f(x, y) \in \mathbb{Q}[x, y]$ be a homogeneous polynomial of degree $d$. Assume that $d$ is odd and that $f$ is not divisible by the $\frac{d+1}{2}$ th power of a linear form. Then $f$ has a properly stable model $F \in \mathbb{Z}[x, y]$ over $\mathbb{Z}$. $F$ is unique up to projective equivalence over $\mathbb{Z}$.

Proof. Let $F$ be a semi-stable model over $\mathbb{Z}$. Since $d / 2$ is not an integer, $F$ is actually properly stable over $\mathbb{Z}$. Thus it is unique by (4.7).

6.2. Norm forms. There is a close connection between semi-stable models of norm forms and ideal class groups. For quadratic extensions such a relationship is classical, see for instance [3, VII.2].

6.2.1. Definition. Let $L \supset \mathbb{Q}$ be a field extension of degree $n$ and $S \subset L$ the ring of algebraic integers. Let $u_{1}, \ldots, u_{n}$ be a $\mathbb{Z}$-basis of $S$ and

$$
N_{L / \mathbb{Q}}(\mathbf{x}):=N_{L / \mathbb{Q}}\left(\sum x_{i} u_{i}\right)
$$

the corresponding norm form (cf. [3, V.1]), viewed as a degree $n$ homogeneous polynomial in the variables $x_{1}, \ldots, x_{n} . N_{L / \mathbb{Q}}(\mathbf{x})$ is unique up to affine equivalence over $\mathbb{Z}$.

If $u_{1}, \ldots, u_{n}$ is any $\mathbb{Q}$-basis of $L$ and $c \in \mathbb{Q}^{*}$, then we obtain a generalized norm form

$$
c \cdot N_{L / \mathbb{Q}}\left(\sum x_{i} u_{i}\right)
$$

A generalized norm form is projectively equivalent over $\mathbb{Q}$ to the norm form of $L / \mathbb{Q}$.

6.2.2. Lemma. A generalized norm form $c \cdot N_{L / K}\left(\sum x_{i} u_{i}\right)$ is semi-stable over $\mathbb{Z}$ iff $u_{1}, \ldots, u_{n}$ is a $\mathbb{Z}$-basis of a fractional $S$-ideal $I \subset L$ and $c= \pm N_{L / \mathbb{Q}}(I)^{-1}$, where $N_{L / \mathbb{Q}}(I)$ is the ideal norm (cf. [3, II.4]).

From this one obtains the following:

6.2.3. Theorem. Let $L \supset \mathbb{Q}$ be a Galois extension with norm form $N_{L / \mathbb{Q}}(\mathbf{x})$. There is a one-to-one correspondence

$$
\left\{\begin{array}{c}
\text { semi-stable models of } N_{L / \mathbb{Q}}(\mathbf{x}) \\
\text { modulo projective equivalence over } \mathbb{Z}
\end{array}\right\} \Leftrightarrow \quad\left\{\begin{array}{c}
\text { ideal class group of } L \\
\text { modulo } \operatorname{Gal}(L / \mathbb{Q})
\end{array}\right\} \text {. }
$$

Similar results hold for non-Galois extensions as well.

6.3. Quadratic forms. Let $q(\mathbf{x})$ be a quadratic form over the $p$-adic field $\mathbb{Q}_{p}$, and assume that $p \neq 2$. The concept of "maximale Gitter" of $[2, \mathrm{p} .50]$ and the notion of " $f$-norm" as defined in [11, II] provide a quadratic form $Q(\mathbf{x})$ over $\mathbb{Z}_{p}$ which is equivalent to $q(\mathbf{x})$ over $\mathbb{Q}_{p}$. The theory of semi-stable models provides a generalization of these, which works in full generality. In this case the results are nicer if we work with affine equivalence.

6.3.1. Notation. Let $R$ be a local Dedekind domain with quotient field $K$, maximal ideal $(p)$ and residue field $k$. Let $q(\mathbf{x}) \in K\left[x_{0}, \ldots, x_{n}\right]$ be a quadratic form such that $(q=0) \subset \mathbb{P}_{K}^{n}$ is a smooth quadric and let $Q(\mathbf{x}) \in R\left[x_{0}, \ldots, x_{n}\right]$ be affine equivalent to $q$.

It should be emphasized that I even allow the case char $K=2$.

For quadratic forms affine semi-stability turns out to be equivalent to the following very simple notion, which we adopt as our definition: 
6.3.2. Definition. $Q$ is affine semi-stable iff there is no change of coordinates $\mathbf{y}=M \mathbf{x}($ where $M \in S L(n+1, R))$ such that $Q\left(p^{-1} y_{0}, y_{1}, \ldots, y_{n}\right) \in R\left[x_{0}, \ldots, x_{n}\right]$.

6.3.3. Theorem. Notation as in (6.3.1). Let $q(\mathbf{x}) \in K\left[x_{0}, \ldots, x_{n}\right]$ be a quadratic form such that $(q=0) \subset \mathbb{P}_{K}^{n}$ is a smooth quadric. Then $q$ has a unique affine semi-stable model $Q \in R\left[x_{0}, \ldots, x_{n}\right]$ (up to affine equivalence over $R$ ).

Moreover, if $Q^{\prime} \in R\left[x_{0}, \ldots, x_{n}\right]$ is equivalent to $q(\mathbf{x})$ over $K$, then there is a matrix $T \in M(n+1, R)$ such that $Q^{\prime}(\mathbf{x})=Q(T \mathbf{x})$.

6.4. Cubic forms. Corti [1] proposed a program of constructing what he calls "standard models" of cubic surfaces over local Dedekind domains [1, Conjectures 2.11 and 2.14]. He established several steps of the program, and his proofs are complete for families of cubic surfaces over a smooth curve in characteristic zero. Although he does not explicitly state it, his conjectures can be easily reformulated for cubic hypersurfaces. Our results imply that his conjectures are true, even for cubic hypersurfaces:

6.4.1. Proposition. Conjectures 2.11 and 2.14 of [1] are true.

The main point of our discussion is, however, the observation, that the models proposed by Corti are not always the optimal ones. In our terminology, he uses only weight systems where all the weights are 0 or 1 . As $[1,5.8]$ shows, this does not give as much uniqueness as (5.2).

At least for cubic surfaces, one needs to use two more weight systems:

6.4.2. Proposition. In order to check semi-stability of a family of cubic surfaces over a one-dimensional regular scheme, it is sufficient to use weight systems with the following five weight sequences:

$$
(0,0,0,1),(0,0,1,1),(0,1,1,1),(0,1,2,2),(0,2,2,3) .
$$

This implies that for cubic forms in four variables over $\mathbb{Z},(4.3)$ becomes an effective algorithm.

6.4.3. Example. Let $R=\mathbb{C}[[t]]$ and consider the cubic form $F_{1}=x_{0}^{3}+x_{1}^{3}+x_{2}^{2} x_{3}+$ $t^{6} x_{3}^{3}$. It is easy to see that it is semi-stable with respect to every weight system where all the weights are 0 or $1 . F_{1}$ is unstable with weights $(2,2,3,0)$, and we obtain the properly stable cubic form $F_{2}=x_{0}^{3}+x_{1}^{3}+x_{2}^{2} x_{3}+x_{3}^{3}$, which is the unique semi-stable model by (5.2). In this example $F_{1}$ is not representable by $F_{2}$, that is, there is no matrix $T \in M(4, \mathbb{C}[[t]])$ such that $F_{1}(\mathbf{x})=F_{2}(T \mathbf{x})$. Thus $F_{1}$ is not representable by any semi-stable model.

\section{REFERENCES}

1. A. Corti, Del Pezzo surfaces over Dedekind schemes, Ann. Math. 144 (1996), 641-683. CMP 97:06

2. M. Eichler, Quadratische Formen und orthogonale Gruppen, Springer Verlag, 1952. MR 14:540a

3. A. Fröhlich and M. Taylor, Algebraic number theory, Cambridge Univ. Press, 1991. MR 94d: 11078

4. J. Humphreys, Arithmetic groups, Springer Lecture Notes 789, 1980. MR 82j:10041

5. J. Kollár, Rational curves on algebraic varieties, Springer Verlag, Ergebnisse der Math. vol. 32, 1996.

6. R. Laxton and D. Lewis, Forms of degree 7 and 11 over p-adic fields, Proc. Symp. Pure Math., vol. 8, pp. 16-21, Amer. Math. Soc., Providence, RI, 1965. MR 31:160 
7. D. Lewis, Diophantine problems: solved and unsolved, Number theory and applications, Kluwer, Dordrecht, 1989, pp. 103-121. MR 92f:11054

8. F. Macaulay, The algebraic theory of modular systems, Cambridge Univ. Press, Cambridge, 1916. MR 95i: 13001

9. D. Mumford and J. Fogarty, Geometric invariant theory, 2nd ed., Springer, 1982. MR 86a: 14006

10. J. Silverman, The arithmetic of elliptic curves, Springer, 1986. MR 87g:11070

11. T. Springer, Quadratic forms over fields with a discrete valuation. I, Indag. Math. 17 (1955), 352-362; II, 18 (1956), 238-246. MR 17:17a; MR 17:945e

12. B. L. van der Waerden, Algebra, vol. I (translated from the 7th German ed.), vol II (translated from the 5th German ed.), Springer-Verlag, New York, 1991. MR 91h:00009a,b

University of Utah, Salt Lake City, UT 84112

E-mail address: kollar@math.utah.edu 